PROCEEDINGS OF THE

AMERICAN MATHEMATICAL SOCIETY

Volume 126, Number 2, February 1998, Pages 545-552

S 0002-9939(98)04059-3

\title{
A WEIGHTED POINCARÉ INEQUALITY WITH A DOUBLING WEIGHT
}

\author{
RITVA HURRI-SYRJĀNEN
}

(Communicated by Palle E. T. Jorgensen)

\begin{abstract}
We show that unbounded John domains (and even a larger class of domains than John domains) satisfy the weighted Poincaré inequality

$$
\inf _{a \in \mathbb{R}}\|u(x)-a\|_{L^{q}\left(D, w_{1}\right)} \leq C\|\nabla u(x)\|_{L^{p}\left(D, w_{2}\right)}
$$

whenever $u$ is a Lipschitz function on $D, w_{1}$ is a doubling weight, and weights satisfy certain cube conditions, and $C=C\left(D, p, q, w_{1}, w_{2}\right)$.
\end{abstract}

\section{INTRODUCTION}

In this note we generalize results considering weighted Poincaré inequalities. My work was stimulated by a paper of Chua [C]. If $D$ is a bounded John domain and if there exists a constant $C_{1}<\infty$ such that the inequality

$$
|Q|^{\frac{1}{n}-1}\left(\int_{Q} w_{1}(x) d x\right)^{1 / q}\left(\int_{Q} w_{2}(x)^{-1 / p-1} d x\right)^{p-1 / p} \leq C_{1}
$$

holds for all Whitney cubes of $D$, then

$$
\left\|u(x)-u_{D, w_{1}}\right\|_{L^{q}\left(D, w_{1}\right)} \leq C_{2}\|\nabla u(x)\|_{L^{p}\left(D, w_{2}\right)}
$$

whenever $w_{1}$ is a doubling weight and $1<p<q<\infty$. If $p=q$, instead of (1.1) we need to require that

$$
|Q|^{1 / n}\left(\frac{1}{|Q|} \int_{Q} w_{1}^{r}(x) d x\right)^{1 / p r}\left(\frac{1}{|Q|} \int_{Q} w_{2}^{-r / p-1}\right)^{p-1 / p r} \leq C_{1}
$$

holds for any $r>1$. These results are implicitly in Chua's paper.

We study the following generalized inequality:

$$
\inf _{a \in \mathbb{R}}\|u(x)-a\|_{L^{q}\left(D, w_{1}\right)} \leq C\|\nabla u(x)\|_{L^{p}\left(D, w_{2}\right)},
$$

where $u$ is a Lipschitz function and $1<p \leq q<\infty$. If $D$ satisfies (1.2), we write $D \in \mathcal{P}(q, p)$ with $w_{1}$ and $w_{2}$ and $C=\mathcal{K}_{q, p}\left(D, w_{1}, w_{2}\right)$. We show that unbounded John domains $D$ satisfy (1.2) whenever (1.1) holds for $1<p<q<\infty$ (respectively $(1.1 *)$ for $p=q)$ and $w_{1}$ is a doubling weight, Theorem 1.8. We also show that there is a larger class than John domains which satisfy (1.1) for $1<p<q<\infty$ (respectively $(1.1 *)$ for $p=q)$ under above conditions, Theorem 1.3. In this manner

Received by the editors January 5, 1996 and, in revised form, August 22, 1996.

1991 Mathematics Subject Classification. Primary 46Exx, 26Dxx.

(C)1998 American Mathematical Society 
a weighted result for so-called rooms and corridors domains is obtained, Example 4.1 .

Our main theorems are Theorems 1.3 and 1.8 .

1.3 Theorem. Let a domain $\mathcal{G}$ be the union of domains $D_{i} \in \mathcal{P}(q, p)$ with a doubling weight $w_{1}$ and a weight $w_{2}$ such that

$$
\mathcal{K}_{q, p}\left(D_{i}, w_{1}, w_{2}\right) \leq C_{0}<\infty, \quad i=1,2, \ldots .
$$

Suppose that each domain $D_{i}$ lies in a cube $Q_{i}$ with the following three properties. There are constants $C_{i}, i=1,2,3$, such that

$$
\sum_{j=1}^{\infty} \chi_{Q_{j}}(x) \leq C_{1} \chi_{\bigcup_{j=1}^{\infty} Q_{j}}(x)
$$

for all $x \in R^{n}$,

$$
Q_{i} \subset C_{2} Q_{j}
$$

where $j=1,2, \ldots, i$, and

$$
\mathcal{K}_{q, p}\left(D_{i}, w_{1}, w_{2}\right)^{q} w_{1}\left(Q_{i}\right) \leq C_{3} \min \left\{w_{1}\left(D_{i} \cap D_{i-1}\right), w_{1}\left(D_{i} \cap D_{i+1}\right)\right\} .
$$

Then $\mathcal{G}$ is a $(q, p)$-Poincaré domain with $w_{1}$ and $w_{2}$.

1.8 Theorem. Let $w_{1}$ be a doubling weight. Suppose that $D$ is an unbounded John domain. Suppose that there exists a constant $C<\infty$ such that the inequality (1.1) for $1<p<q<\infty$ (respectively $(1.1 *)$ for $p=q$ ) holds for all cubes $Q \subset D$. Then $D$ is a weighted $(q, p)$-Poincaré domain with $w_{1}$ and $w_{2}$.

The proofs for Theorem 1.3 and Theorem 1.8 are given in $\S 3$. An example and corollaries in $\S 4$ reveal that we have generalized results of Chao et al. [CWZ], Evans and Harris [EH], and Hurri $[\mathrm{H}]$.

\section{Preliminaries}

Notation. Throughout this paper we let $D$ be a domain of euclidean $n$-space $R^{n}$, $n \geq 2$. We suppose that $1 \leq p \leq q<\infty$ unless otherwise stated.

The diameter of a set $A$ is written as $\operatorname{dia}(A)$. We write $t Q$ for the cube with the same center as $Q$ and dilated by a factor $t>1$.

We let $C(*, \cdots, *)$ denote a constant which depends only on the quantities appearing in the parentheses.

A weight (function) is a nonnegative measurable function on $R^{n}$. A weight $w$ is a doubling weight (that is, $w$ satisfies a doubling condition) if there exists a constant $t<\infty$ such that

$$
\int_{2 Q} w(x) d x \leq t \int_{Q} w(x) d x \text { for all cubes } Q \subset R^{n} .
$$

$A_{p}$-weights are doubling weights.

The average of a function $u$ over a domain $D$ with finite Lebesgue measure $|D|$ is

$$
u_{D}=\frac{1}{|D|} \int_{D} u(x) d x
$$


and with an arbitrary weight $w$

$$
u_{D, w}=\frac{1}{\int_{D} w(x) d x} \int_{D} u(x) w(x) d x
$$

whenever

$$
0<\int_{D} w(x) d x<\infty
$$

We write

$$
\|u\|_{L^{p}(D, w)}=\left(\int_{D}|u(x)|^{p} w(x) d x\right)^{1 / p}
$$

where $u$ is a Lipschitz function on $D$. The distributional gradient is written as $\nabla u=\left(\partial_{1} u, \ldots, \partial_{n} u\right)$.

We recall a lemma due to Strömberg and Wheeden.

2.1 Lemma ([StW, Lemma 2.3], [C, Lemma 2.5]). Let $\left\{Q_{\alpha}\right\}_{\alpha \in \mathcal{I}}$ be an arbitrary family of cubes in $R^{n}$. If $\left\{a_{\alpha}\right\}_{\alpha \in \mathcal{I}}$ is a family of nonnegative real numbers and $w$ is a doubling weight, then for $1 \leq p<\infty$ and $N \geq 1$ we have

$$
\left\|\sum_{\alpha} a_{\alpha} \chi_{N Q_{\alpha}}\right\|_{L^{p}\left(R^{n}, w\right)} \leq C(n, p, N, w)\left\|\sum_{\alpha} a_{\alpha} \chi_{Q_{\alpha}}\right\|_{L^{p}\left(R^{n}, w\right)} .
$$

The Hölder inequality and the Minkowski inequality yield the following useful lemma.

2.2 Lemma. Let $D$ be a domain and $A \subset D$ be a set such that $\int_{A} w(x) d x<\infty$. Then for each $a \in R$

$$
\left\|u-u_{A, w}\right\|_{L^{p}(D, w)} \leq 2\left(\frac{\int_{D} w(x) d x}{\int_{A} w(x) d x}\right)^{1 / p}\|u-a\|_{L^{p}(D, w)}
$$

where $u$ is a Lipschitz function on D.

$(q, p)$-Poincaré domains with weights $w_{1}$ and $w_{2}$. Let $D \subset R^{n}$ be a domain and let $1 \leq p \leq q<\infty$. Let $w_{1}$ and $w_{2}$ be weight functions. If there exists a constant $\mathcal{K}=\mathcal{K}\left(D, p, q, w_{1}, w_{2}\right)<\infty$ such that the inequality

$$
\inf _{a \in R}\|u-a\|_{L^{q}\left(D, w_{1}\right)} \leq \mathcal{K}\|\nabla u\|_{L^{p}\left(D, w_{2}\right)}
$$

holds for all Lipschitz functions $u$, then $D$ is a $(q, p)$-Poincaré domain with weights $w_{1}$ and $w_{2}$. We write $D \in \mathcal{P}(q, p)$ with $w_{1}$ and $w_{2}$.

John domains. Let $E$ be a closed arc with endpoints $a$ and $b$. The subarc between $x$ and $y$ is denoted by $E[x, y]$. For $x$ in $E \backslash\{a, b\}$ write

$$
q(x)=\min \{\operatorname{dia}(E[a, x]), \operatorname{dia}(E[b, x])\} .
$$

Let $c \geq 1$. A domain $D$ in $R^{n}$ is a $c$-John domain, if each pair of distinct points $a$ and $b$ in $D$ can be joined by an $\operatorname{arc} E$ such that

$$
\operatorname{cig} E(a, b)=\bigcup\left\{B\left(x, \frac{q(x)}{c}\right) \mid x \in E \backslash\{a, b\}\right\} \subset D .
$$

Balls, convex domains, domains with smooth boundaries are John domains as well as a snowflake domain. 
Bojarski proved that a bounded $b$-John domain satisfies the $(q, p)$-Poincaré inequality with $w_{1}=w_{2}=1\left[\mathrm{~B}\right.$, Chapter 6]. Unbounded John domains are $\left(\frac{n p}{n-p}, p\right)$ Poincaré domains with $w_{1}=w_{2}=1$ [H-S, Corollary 4.6].

We recall the following lemma due to Väisa̋lä.

2.4 Lemma ([V, Theorem 4.6]). Let $D$ be an unbounded b-John domain. Then there are $b_{0}$-John domains $D_{i}$ such that $D_{i} \subset \bar{D}_{i} \subset D_{i+1}, i=1,2, \ldots$, and $D=$ $\bigcup_{i=1}^{\infty} D_{i}$.

Sawyer and Wheeden have given several sufficient and necessary conditions for weights $w_{1}$ and $w_{2}$ so that a cube is a weighted $(q, p)$-Poincaré domain. We write down here one of their theorems which was refined by Chua for a doubling weight $w_{1}$.

2.5 Lemma ([C, Theorem 2.14]). Suppose that $w_{1}$ is a doubling weight. Then for all Lipschitz functions $u$ and a cube $Q_{0}$

$$
\left\|u-u_{Q_{0}, w_{1}}\right\|_{L^{q}\left(Q_{0}, w_{1}\right)} \leq \mathcal{K}_{q, p}\|\nabla u\|_{L^{p}\left(Q_{0}, w_{2}\right)}
$$

where

$$
\mathcal{K}_{p, q}=\mathcal{K}_{q, p}\left(w_{1}\right) \sup _{Q \subset Q_{0}}|Q|^{\frac{1}{n}-1}\left(\int_{Q} w_{1}(x) d x\right)^{1 / q}\left(\int_{Q} w_{2}(x)^{-1 / p-1} d x\right)^{p-1 / p}
$$

whenever $p<q$.

$$
\text { If } p=q \text {, then }
$$

$$
\left\|u-u_{Q_{0}, w_{1}}\right\|_{L^{p}\left(Q_{0}, w_{1}\right)} \leq \mathcal{K}_{p}\|\nabla u\|_{L^{p}\left(Q_{0}, w_{2}\right)}
$$

with

$$
\mathcal{K}_{p}=\mathcal{K}_{p}\left(r, w_{1}\right) \sup _{Q \subset Q_{0}}|Q|^{1 / n}\left(f_{Q} w_{1}(x)^{r} d x\right)^{1 / p r}\left(f_{Q} w_{2}(x)^{-r /(p-1)} d x\right)^{(p-1) / p r}
$$

for any $r>1$.

Proof. Let $f=u-u_{Q_{0}, w_{1}}$ in [C, Theorem 2.14].

Chua considered Boman's chain condition domains $[\mathrm{C}]$. An especial case of his theorem [C, Theorem 1.5] is the following lemma.

2.6 Lemma. Let $D$ be a bounded $b_{0}$-John domain and let $w_{1}$ be a doubling weight. Suppose that there exists a constant $C<\infty$ such that for all cubes $Q$ in $D$ (1.1) holds whenever $1<p<q<\infty$ (respectively, $(1.1 *)$ holds for $p=q$ ).

Then

$$
\left\|u-u_{D, w_{1}}\right\|_{L^{q}\left(D, w_{1}\right)} \leq \mathcal{K}_{p, q}\left(b_{0}, C, w_{1}\right)\|\nabla u\|_{L^{p}\left(D, w_{2}\right)} .
$$

Proof. Lemma 2.5 and [C, Theorem 1.5].

Proof for Theorem 1.3. By the given decomposition of $\mathcal{G}$

$$
\begin{aligned}
& \int_{\mathcal{G}}\left|u(y)-u_{D_{1}, w_{1}}\right|^{q} w_{1}(y) d y \leq \sum_{i=1}^{\infty} \int_{D_{i}}\left|u(y)-u_{D_{1}, w_{1}}\right|{ }^{q} w_{1}(y) d y \\
& \quad \leq 2^{q-1}\left(\sum_{i=1}^{\infty} \int_{D_{i}}\left|u(y)-u_{D_{i}, w_{1}}\right|{ }^{q} w_{1}(y) d y+\sum_{i=1}^{\infty} \int_{D_{i}}\left|u_{D_{i}, w_{1}}-u_{D_{1}, w_{1}}\right|{ }^{q} w_{1}(y) d y\right)
\end{aligned}
$$


Since $D_{i} \in \mathcal{P}(q, p)$ with weights $w_{1}$ and $w_{2}$ and $\mathcal{K}_{q, p}\left(D_{i}, w_{1}, w_{2}\right) \leq C_{0}$, we obtain

$$
\begin{aligned}
& \sum_{i=1}^{\infty} \int_{D_{i}}\left|u(y)-u_{D_{i}, w_{1}}\right|^{q} w_{1}(y) d y \\
& \quad \leq \sum_{i=1}^{\infty}\left(\mathcal{K}_{q, p}\left(D_{i}, w_{1}, w_{2}\right)\left(\int_{D_{i}}|\nabla u(y)|^{p} w_{2}(y) d y\right)^{1 / p}\right)^{q} \\
& \quad \leq C_{0}^{q}\left(\int_{\mathcal{G}}|\nabla u(y)|^{p} w_{2}(y) d y\right)^{q / p} .
\end{aligned}
$$

To estimate the second sum we use the triangle inequality and the weighted $(q, p)$ Poincaré inequality in $D_{i}$. First,

$$
\begin{aligned}
& \left|u_{D_{j}, w_{1}}-u_{D_{j+1}, w_{1}}\right|^{q} \\
& \quad=\frac{1}{w_{1}\left(D_{j} \cap D_{j+1}\right)} \int_{D_{j} \cap D_{j+1}}\left|u_{D_{j}, w_{1}}-u_{D_{j+1}, w_{1}}\right|{ }^{q} w_{1}(x) d x \\
& \quad \leq \frac{2^{q-1}}{w_{1}\left(D_{j} \cap D_{j+1}\right)} \sum_{h=j}^{j+1} \mathcal{K}_{q, p}^{q}\left(D_{h}, w_{1}, w_{2}\right)\left(\int_{D}|\nabla u(x)|^{p} w_{2}(x) d x\right)^{q / p} .
\end{aligned}
$$

Hence, the triangle inequality, the condition (1.7), and the engulfing property,

$$
D_{i} \subset Q_{i} \subset C_{2} Q_{j}, \quad j=1,2, \ldots, i,
$$

yield that

$$
\begin{aligned}
& \sum_{i=1}^{\infty} \int_{D_{i}}\left|u_{D_{i}, w_{1}}-u_{D_{1}, w_{1}}\right|^{q} w_{1}(x) d x \\
& \quad \leq \sum_{i=1}^{\infty} \int_{D_{i}}\left(\sum_{j=1}^{i-1}\left|u_{D_{j}, w_{1}}-u_{D_{j+1}, w_{1}}\right| \chi_{D_{i}}(x)\right)^{q} w_{1}(x) d x \\
& \quad \leq C_{4} \int_{R^{n}}\left(\sum_{j=1}^{\infty} \frac{1}{w_{1}\left(Q_{j}\right)^{1 / q}}\left(\int_{D_{j}}|\nabla u(y)|^{p} w_{2}(y) d y \chi_{C_{2} Q_{j}}(x)\right)^{1 / p}\right)^{q} w_{1}(x) d x .
\end{aligned}
$$

Lemma 2.1 implies

$$
\begin{aligned}
& \sum_{i=1}^{\infty} \int_{D_{i}}\left|u_{D_{j}, w_{1}}-u_{D_{j+1}, w_{1}}\right|{ }^{q} w_{1}(x) d x \\
& \quad \leq C_{5} \int_{R^{n}}\left(\sum_{j=1}^{\infty} \frac{1}{w_{1}\left(Q_{j}\right)^{1 / q}}\left(\int_{D_{j}}|\nabla u(y)|^{p} w_{2}(y) d y \chi_{Q_{j}}(x)\right)^{1 / p}\right)^{q} w_{1}(x) d x \\
& \quad \leq C_{6} \sum_{j=1}^{\infty} \frac{1}{w_{1}\left(Q_{j}\right)}\left(\int_{D_{j}}|\nabla u(y)|^{p} w_{2}(y) d y\right)^{q / p} \int_{R^{n}} \chi_{Q_{j}}(x) w_{1}(x) d x \\
& \quad \leq C_{7}\left(\int_{\mathcal{G}}|\nabla u(y)|^{p} w_{2}(y) d y\right)^{q / p} .
\end{aligned}
$$


Proof for Theorem 1.8. Since $D$ is an unbounded John domain, there are $b_{0}$-John domains $D_{i} \subset \bar{D}_{i} \subset D_{i+1}$ such that $D=\bigcup_{i=1}^{\infty} D_{i}$ by Lemma 2.4.

We set

$$
u_{i}=\frac{1}{\int_{D_{i}} w_{1}(x) d x} \int_{D_{i}} u(x) w_{1}(x) d x .
$$

We will use $D_{1}$ to obtain for $\left|u_{i}\right|$ an upper bound which does not depend on $i$. The triangle inequality yields

$$
\begin{aligned}
\left|u_{i}\right| & =\left(\int_{D_{1}} w_{1}(x) d x\right)^{-1} \int_{D_{1}}\left|u_{i}\right| w_{1}(x) d x \\
& \leq\left(\int_{D_{1}} w_{1}(x) d x\right)^{-1}\left(\int_{D_{1}}\left|u(x)-u_{i}\right| w_{1}(x) d x+\int_{D_{1}}|u(x)| w_{1}(x) d x\right)
\end{aligned}
$$

where we may assume that

$$
0<\int_{D_{1}} w(x) d x<\infty \text { and } \int_{D_{1}}|u(x)| w_{1}(x) d x<\infty .
$$

By Lemma 2.6

$$
\begin{aligned}
\int_{D_{i}}\left|u(x)-u_{i}\right| w_{1}(x) d x & \leq\left(\int_{D_{1}} w_{1}(x) d x\right)^{1-\frac{1}{q}}\left\|u-u_{i}\right\|_{L^{q}\left(D_{i}, w_{1}\right)} \\
& \leq\left(\int_{D_{1}} w_{1}(x) d x\right)^{1-\frac{1}{q}}\left\|u-u_{i}\right\|_{L^{q}\left(D_{i}, w_{1}\right)} \\
& \leq\left(\int_{D_{1}} w_{1}(x) d x\right)^{1-\frac{1}{q}} \mathcal{K}_{q, p}\left(b_{0}, C, w_{1}\right)\|\nabla u\|_{L^{p}\left(D, w_{2}\right)} .
\end{aligned}
$$

Thus $\left(u_{i}\right)$ is a bounded sequence and hence there exists a convergent subsequence $\left(u_{i j}\right)$ and $b \in R$ such that $\lim _{j \rightarrow \infty} u_{i j}=b$.

Since

$$
\lim _{j \rightarrow \infty} \chi_{D_{j}}(x)\left|u(x)-u_{j}\right|^{q}=\chi_{D}(x)|u(x)-b|^{q},
$$

Fatou's lemma and Lemma 2.6 yield that

$$
\begin{aligned}
\int_{D}|u(x)-b|^{q} w_{1}(x) d x & =\int_{D} \lim _{j \rightarrow \infty} \chi_{D_{j}}(x)\left|u(x)-u_{j}\right|^{q} w_{1}(x) d x \\
& \leq \underline{\lim }_{j \rightarrow \infty} \int_{D} \chi_{D_{j}}(x)\left|u(x)-u_{j}\right|^{q} w_{1}(x) d x \\
& \leq \varliminf_{j \rightarrow \infty}\left(\left(\mathcal{K}_{q, p}\left(b_{0}, C, w_{1}\right) \int_{D_{j}}|\nabla u(x)|^{p} w_{2}(x) d x\right)^{1 / p}\right)^{q} \\
& \leq \underline{\lim }_{j \rightarrow \infty}\left(\mathcal{K}_{q, p}\left(b_{0}, C, w_{1}\right) \int_{D}|\nabla u(x)|^{p} w_{2}(x) d x\right)^{q / p} \\
& =\left(\mathcal{K}_{q, p}\left(D, w_{1}\right) \int_{D}|\nabla u(x)|^{p} w_{2}(x) d x\right)^{q / p} .
\end{aligned}
$$




\section{FURTHER REMARKS}

Example 4.1 considers the rooms and corridors domain.

4.1 Example. Let $G=\bigcup_{i=1}^{\infty} D_{i}$ be a domain where the sets $D_{i}, i=1,2, \ldots$, are defined as follows: Let $\left(h_{i}\right)$ and $\left(\delta_{2 i}\right)$ be sequences such that $h_{i}=M^{-i}, M>1$, and $\delta_{2 i}=b M^{-2 a i}, b>0, a>1$. We set $\eta_{2 i}=M^{-2(i+1)}, i=1,2, \ldots$, and $\sum_{i=1}^{k} h_{i}=d_{k}$. We define

$$
\begin{gathered}
D_{2 i-1}=\left(d_{2 i-1}-h_{2 i-1}, d_{2 i-2}\right) \cdot\left(-\frac{1}{2} h_{2 i-1}, \frac{1}{2} h_{2 i+1}\right)^{n-1}, \\
D_{2 i}=\left(d_{2 i-1}-\frac{1}{2} \eta_{2 i}, d_{2 i-1}+h_{2 i}+\frac{1}{2} \eta_{2 i}\right) \cdot\left(-\frac{1}{2} \delta_{2 i}, \frac{1}{2} \delta_{2 i}\right)^{n-1} .
\end{gathered}
$$

By $[\mathrm{H}] G$ is an ordinary Poincaré domain, if and only if $p \geq(n-1)(a-1)$.

Adjoin the cubes $Q_{i}$ to the sets $D_{i}$ as follows: $Q_{2 i-1}=D_{2 i-1}$ and

$$
Q_{2 i}=\left(d_{2 i-1}-\frac{1}{2} \eta_{2 i}, d_{2 i-1}+h_{2 i}+\frac{1}{2} \eta_{2 i}\right) \cdot\left(-\frac{1}{2}\left(h_{2 i}+\eta_{2 i}\right), \frac{1}{2}\left(h_{2 i}+\eta_{2 i}\right)\right)^{n-1} .
$$

We choose $w_{1}(x)=1$ and $w_{2}(x)=d(x, \partial G)^{\alpha p}$. To check (1.7) we need the fact that the weighted Poincaré constant in this case is $|D|^{q+\frac{1-\alpha}{n}-\frac{1}{p}} c^{n}$ for a $c$-John domain by [H-S2]. Using this we obtain that weighted Poincaré inequality (1.3) holds whenever

$$
n\left(2-a-\frac{q}{p}+p(1-a)\right)+q(1-\alpha)-1+a \geq 0 .
$$

This generalizes the results of $[\mathrm{EH}]$ and $[\mathrm{H}]$ to the weighted case.

The proof for Theorem 1.8 has the following interesting corollaries.

4.2 Corollary. Let $w_{1}$ be a doubling weight. Suppose that $D$ is an unbounded John domain. Suppose that there exists a constant $C<\infty$ such that for all cubes $Q \subset D$ the inequality (1.1) holds whenever $1<p<q<\infty$ (respectively, $(1.1 *)$ for $p=q$ ). Then there is a constant $\mathcal{K}_{q, p}\left(w_{1}\right)<\infty$ such that the inequality

$$
\|u\|_{L^{q}\left(D, w_{1}\right)} \leq \mathcal{K}_{q, p}\left(D, w_{1}\right)\|\nabla u\|_{L^{p}\left(D, w_{2}\right)}
$$

holds for all Lipschitz functions u.

Proof. Note that

$$
u_{j}=\frac{1}{\int_{D_{j}} w_{1}(x) d x} \int_{D_{j}} u(x) w_{1}(x) d x \rightarrow 0
$$

when we assume that $\int_{D} u(x) w_{1}(x) d x<\infty$. This follows from the proof for Theorem 1.8 .

An especial case of Corollary 4.2 is the following result.

4.3 Corollary. If $D$ is an unbounded John domain then there exists a constant $C=C(n, p)<\infty$ such that

$$
\|u\|_{L^{n p / n-p}(D)} \leq C\|\nabla u\|_{L^{p}(D)}
$$

holds for all Lipschitz functions $u$. 
Corollary 4.3 generalizes the result of Chen et al. [CWZ] who showed that (4.4) holds for domains with a cone condition.

\section{REFERENCES}

[B] Bojarski, B., Remarks on Sobolev imbedding inequalities, Complex Analysis (Joensuu 1987), Lecture Notes in Math., Springer-Verlag, Berlin and Heidelberg 1351 (1988), 5268. MR 90b:46068

[C] Chua, S.-K., Weighted Sobolev inequalities on domains satisfying the chain condition, Proc. Amer. Math. Soc. 117 (1993), 449-457. MR 93d:46050

[CWZ] Chen, Z.Q., R.J. Williams and Z. Zhao, A Sobolev inequality and Neumann heat kernel estimate for unbounded domains, Math. Research Letters 1 (1994), 177-184. MR 90d:70034

[EH] Evans, W.D. and D.J. Harris, Sobolev embeddings for generalized ridged domains, Proc. London Math. Soc. 54 (3) (1987), 141-175. MR 88b:46056

[H] Hurri, R., Poincaré domains in $R^{n}$, Ann. Acad. Sci. Fenn. Ser. IA, Dissertations 71 (1988), 1-41. MR 90a:30074

[H-S] Hurri-Syrjānen, R., Unbounded Poincaré domains, Ann. Acad. Sci. Fenn. Ser. IA 17 (1992), 409-423. MR 93k:46022

[H-S2] Hurri-Syrjānen, R., An improved Poincaré inequality, Proc. Amer. Math. Soc. 120 (1994), 213-222. MR 94b:46047

[SW] Sawyer, E. and R.L. Wheeden, Weighted inequalities for fractional integrals on Euclidean and homogeneous spaces, Amer. J. Math. 114 (1992), 813-874. MR 94i:42024

[StW] Strömberg, J.-O. and R.L. Wheeden, Fractional integrals on weighted $H^{p}$ and $L^{p}$ spaces, Trans. Amer. Math. Soc. 287 (1985), 293-321. MR 86f:42016

[V] Va̋isa̋lä, J., Exhaustions of John domains, Ann. Acad. Sci. Fenn. Ser. IA 19 (1994), 47-54. MR 94i:30024

Department of Mathematics, University of Texas, Austin, Texas 78712

E-mail address: syrjanen@math.utexas.edu

Current address: Department of Mathematics, P.O. Box 4, FIN-00014 University of Helsinki, Finland

E-mail address: hurrisyr@helsinki.fi 